\title{
Providing Universal Location Services Using a Wireless E911 Location Network
}

\author{
James M. Zagami and Steen A. Parl, Signatron Technology Corporation \\ Julian J. Bussgang, Consultant \\ Karen Devereaux Melillo, University of Massachusetts Lowell
}

\begin{abstract}
This article reviews the issues associated with the development $\triangle \mathrm{BSIRACT}$ of an E911 location network in light of the desire to leverage the E911 location network to provide universal non-cellular location-based services. The key issues are illustrated with a system originally developed for locating elderly patients with Alzheimer's disease.
\end{abstract}

planting IS-54), and the European-developed G lobal System for M obile Communications (GSM ). Each of these standards uses different transmitted waveforms and control channel

n J une 1996, the FCC adopted FCC D ocket 94-102 [1] as an official report and order ( $R \& 0)$, thereby officially mandating that wireless 911 callers receive the same level of emergency service as that available to wireline callers who have benefited from wireline E nhanced 911 (E 911). O ne of the key provisions of FCC 94-102 is that cellular, personal communications services (PCS), and specialized mobile radio (SM R) service providers deploy a means of automatically locating 911 callers to within $125 \mathrm{~m}$ in 67 percent of all measurements by 0 ctober 31, 2001. In the process, the FCC R\&O will create what is forecast to be a multibillion-dollar market for caller location technologies [2], which in turn is expected to catalyze the offering of a new array of noncellular location-based services such as monitoring the location of people, cargo, or vehicles.

In this article we review the issues associated with E 911 location services and discuss how a system originally developed by Signatron T echnology Corporation (ST C) for locating lost patients may offer performance and cost advantages for an E 911 location implementation, particularly when the location base station equipment is used to provide both E 911 and general location services.

We begin with a brief overview of the requirements of a cellular E 911 location system and an overview of existing location technologies. We then give an overview of the Signatron Location System (SLS) developed by STC, and conclude with two examples describing how this technology may be applied to locating wandering A lzheimer's disease patients and tracking parolees.

\section{E911 LOCATION NETWORK REQUIREMENTS}

Cellular telephone systems are all similar in their basic architecture. A network of radio base stations that handles multiple callers is controlled by a network of mobile switching centers (M SCs) which are linked via the public telephone network. A typical example is shown in $\mathrm{Fig}$. 1. The home location register $(H L R)$ and vistor location register (VLR) databases maintained at the M SC are used to validate mobile units, locate roaming units, and provide billing records for the central office. While the general architecture of cellular phone networks is similar, the air interfaces differ greatly among cellular standards. In the U nited States, the most common air interface in use is still the A dvanced M obile Phone System (A M PS), but new digital transmission technologies are expected to supplant the A M PS standard over the next decade. The most common digital cellular/PCS technologies are code-division multiple access, or CDMA (EIA/TIA IS-95), time-division multiple access, or TDMA (EIA/TIA IS-136, supdata formats, both of which will generally impact the location system design. As digital systems become operational, a major issue will be the operation of location systems at digital/analog system boundaries where the handset may be in digital mode, and the location measurement may involve the use of both analog and digital receiver sites which may even be in different frequency bands; such operation requires careful design of measurement equipment and coordination among location receivers.

Location technologies that have been proposed to date to meet FCC 94-102 fall into two broad categories: network-based solutions and handset-based solutions.

Proposed network based location systems are generally either independent overlay systems or technologies designed to be integrated into base stations to measure certain signal characteristics, such as time of arrival (TOA) or angle of arrival ( $O$ OA) of the desired transmitted signal as received at several receiver stations from which a location is estimated; there are no modifications to the mobile handsets.

$\mathrm{H}$ andset-based techniques rely on a modified handset to calculate its own position. O ne such technique is to use a G lobal Positioning System (GPS) receiver embedded in the handset. In addition to self-location, these techniques also require a return data path to report the location to the network for relay to the emergency operator. The obvious drawbacks of handset-based approaches are the cost of developing a suitable low-power and economical integrated technology for use in the handsets and the cost of deploying new handsets. Because of the drawbacks of nonnetwork technologies, cellular carriers generally favor the use of a network-based approach, provided the necessary infrastructure is not prohibitively expensive.

A network-based location overlay system can be implemented by deploying location receiver/processors at either existing base stations or new receiver sites. These location receiver/processors are used to capture signals from the desired mobile unit and to transmit either the captured digitized signal or certain signal attributes such as AOA to a computer for location estimation. Preferably, the location receivers will be located at existing base station sites and will use the existing cellular antennas and R F front-end circuitry. There are, however, a number of technical hurdles relating to the level of integration or interaction the location system requires with the existing cellular base stations or switches. In particular, the location system would ideally interface to the existing base stations such that the base station controller can invoke the location system when a 911 call is placed and inform the location system which channel the 911 call has been assigned. In general, a single such interface to base stations pro- 
duced by many different manufacturers is not possible, especially for already deployed A M PS systems. The location system must therefore include a means of monitoring the control channel traffic to detect 911 calls and to decode the subsequently assigned voice channels for location tracking. It is anticipated that digital base station equipment manufacturers will eventually integrate location technology into base stations or provide an interface to the control channel information for selected location systems.

A fter the location system locates the E 911 mobile unit, the location and phone ID must be relayed to the Public Service A nswering Point (PSA P) for display and use by the emergency operator or for automated dispatch of response personnel. This issue is being addressed by the $\mathrm{N}$ ational E mergency N umber A ssociation (NENA) in the form of a standard data format for reporting A utomatic L ocation I dentification $(A L I)$ data and should be followed by prospective E 911 A L I equipment vendors.

In short, we summarize the desired features of a cellular E 911 A L I system as follows:

- M eet FCC 94-102 ( <125 m accuracy for 67 percent of measurements).

- Coverage at least comparable to the cellular system.

- Seamless integration with existing base stations, no modifications to nor interaction with existing base station equipment, other than gaining access to the necessary antenna signals. H owever, some interaction may be required to identify 911 calls in encrypted digital systems.

- M inimal data rate backhaul requirements, minimizing recurring charges.

- Low-cost location receiver equipment.

- U se existing cellular antennas and shelters where possible to avoid added recurring operation costs.

- R eliable operation.

- Expandable for increased future capacity.

- Support for other location services with no impact on cellular capacity nor interaction with cellular systems.

- Capable of interfacing to E 911 terrestrial networks for A LI reporting.

Of course, some approaches may sacrifice one or more of these criteria in order to gain an advantage in another. For instance, A O A approaches generally propose using additional or custom antennas, sacrificing added recurring costs in return for better performance. TDOA approaches can use the existing cellular antennas, but generally require a high-data-rate backhaul. The SL S approach discussed below draws from the benefits of both approaches; the result is that the existing cellular antennas can be used without the need for costly high-speed data backhauls.

\section{OVERVIEW OF POSITION LOCATION TECHNIQUES}

The two most widely known network-based location technologies used in proposed mobile phone location systems are TDOA and A OA. TDOA systems use the principle that the emitter location can be estimated by intersection of the hyperbolae of constant differential TOA s of the signal at two or more pairs of base stations. AOA systems use simple triangulation based on estimated A OA of a signal at two or more base stations to estimate the location of a desired transmitter. $V$ ariations of both of these approaches have been proposed for cellular location and are discussed in [3]. B elow we discuss key characteristics relevant to both cellular phone and noncellular applications.

Conventional TDOA technologies require that the desired signal be captured and digitized at three or more base stations, time-

tagged, and transmitted to a central processor for cross-correlation and position estimation using hyperbolic intersection. Because of system inaccuracies and differences in propagation channels to each of the base stations, the hyperbolae generally will not intersect at the actual location. A Iso, two hyperbolae can intersect at two points, creating ambiguities. The main drawback of TDOA is that a large bandwidth is required to transmit the captured digitized waveform to a central site. This is not only costly, but will also limit the capability of such a system to serve high-capacity commercial location applications. To overcome this, some systems use TOA, where the TOA s of a known signal are estimated directly at each base station. A nother concern for TOA/TDOA methods is that, in rural areas, cellular base stations are widely spaced and often the minimum required three base stations cannot all receive the desired signal. In such areas, additional TDOA location receivers may have to be sited to provide adequate coverage.

A OA systems use antenna arrays consisting of two or more elements which are used to measure the line of bearing as seen at two or more base stations. The location is estimated by triangulation, using a best fit approximation of the intersection of the lines of bearing, or by other methods that try to improve the accuracy of the estimate. The accuracy improves with the size of the array, which implies using either a large number of elements or, in order to lower costs, a large interelement spacing. Since wider antenna spacing introduces ambiguities in the results, the existing cellular diversity antennas cannot be used in traditional A O A location systems. A Iso, A O A systems generally discard other useful signal information, such as amplitude, and thus result in less than optimum accuracy. A OA processing can be less costly than TDOA processing, but the savings in processing cost are generally offset by the fixed and recurring costs of placing custom antenna arrays on an antenna structure for proper operation. A OA systems are particularly susceptible to strong angular multipath degradation and also exhibit very poor performance in rural areas where the linear orientation of base stations along major roads causes geometric dilution of precision (GDOP). The SL S approach is less sensitive to GDOP than either AOA or TDOA alone, since it incorporates AOA and TDOA in position estimation.

A comparison of various location technologies, including the new STC SL S system discussed below, is given in Table 1.

\section{THE SLS APPROACH AND ITS APPLICATIONS}

A number of location technologies are being designed specifically to locate cellular telephones, and some of these technologies may later be adapted to provide additional location services. STC on the other hand, has developed the SL S, a low-cost low-power approach designed specifically for locating or tracking people and objects outfitted with a very-low-cost radio transceiver. The technology relies on a maximum likelihood estimate ( $M L E$ ) of loca- 
tion to achieve higher accuracy than the conventional TOA/TDOA and AOA methods described above. This new SLS technique (patent pending) is also being applied to develop a product that promises to economically meet $\mathrm{E} 911$ requirements so that the same network can be used for a range of low-cost high-capacity location-based services. The SLS processes the wireless signal in such a way that both the direction and differential range of the mobile unit relative to the base stations are taken into account, but without incurring the information loss of

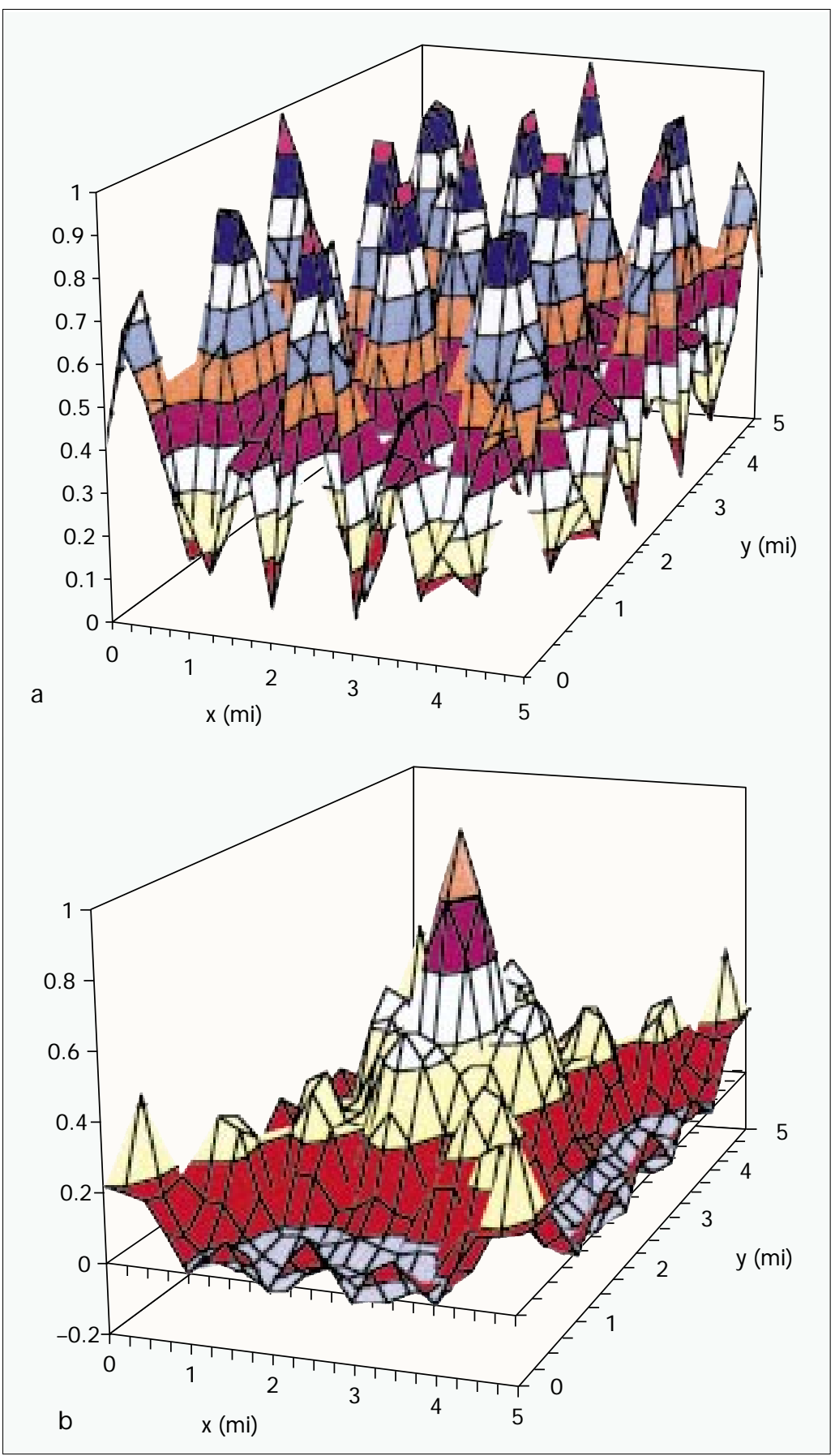

Figure 2. a) A high accuracy likelihood function showing narrow location peaks but many ambiguous locations (e.g., using A $O A$ with a large element spacing); b) the SLS combines high-accuracy high-ambiguity implicit AOA data with low-ambiguity lower-accuracy implicit TDOA data to get the best of both: high accuracy and low ambiguity. the traditional intermediate step of estimating direction angles or time delays.

By utilizing a known signal structure and intelligent assignment of unused cellular channels, the SLS can use existing cellular antennas to simultaneously support both E 911 and noncellular applications, such as the lost patient location application discussed below.

A major benefit of the STC SLS is that the AOA ambiguity associated with using the large diversity antenna spacing at cellular base stations is eliminated by using the implicit TDOA information without adding custom antennas. A nother major benefit is that only very little information need be transmitted to a central site. As an illustration of the former benefit, Fig. 2a shows the likelihood function expected using A O A information only, illustrating how high accuracy unfortunately also means high ambiguity when only two widely spaced antenna elements are used. Figure $2 \mathrm{~b}$ shows that $\mathrm{SL} S$, by incorporating TDOA information, achieves both a low location error (narrow peak of the likelihood function) and low ambiguity (single peak). These typical examples were computer-generated assuming a $100 \mathrm{~mW}$ mobile transmitter and four base stations forming a square with $5 \mathrm{mi}$ sides. A s with any method, there is a finite probability that noise can cause a smaller peak to be erroneously chosen. H owever, such an error can usually be detected by correlating with previous results or requesting a new measurement.

The approach to multipath is twofold: take into account amplitude information, and use more than the minimum number of base station measurements. This approach, while not optimal, is a cost-effective means to meet F CC E 911 requirements. Simulations have demonstrated the SLS method to be relatively insensitive to multipath, and laboratory simulator tests and field tests are planned to verify these results.

We are currently studying the performance of the SL $S$ in rural areas where the geometry of base stations is likely to impair performance. Thus far, computer simulations indicate that SL S performance will exceed that of traditional AOA or TDOA approaches in such areas, providing approximately $75 \mathrm{~m}$ root mean square ( $\mathrm{rms}$ ) error.

\section{NoncelluLAR Applications OF THE SLS}

If an infrastructure deployed to locate cellular phones is to provide additional revenue-generating location services, the requirements of these "other" services must be well understood by the equipment and network developers. It is critical, therefore, that wireless E 911 location technologies and systems take into account the requirements of non-911 services. Cellular carriers must thus focus on deploying a satisfactory and economical solution that combines compliance with FCC 94-102 with support of these other location services. For example, a location system designed to locate a cellular telephone waveform and operate in the cellular network may not necessarily be the best solution for providing the high capacity, wide coverage, accuracy, low cost, and long battery life that will be demanded by many noncellular location service applications.

SLS addresses the fundamental problem of location monitoring, which is defined as the remote radio location of the position of a mobile transmitter. The person or object to be located or tracked carries a very small 
and inexpensive low-power unit, which on command transmits a signal generated by very simple low-cost electronics. The signal is received at several base stations, and the location estimate is found by using a maximum likelihood algorithm, incorporating TDOA and AOA as special cases. In all but a few degenerate cases, only two base stations can suffice. A very inexpensive low-power single integrated circuit (IC) will enable low-cost location devices with many applications, as shown in $\mathrm{Fig}$. 3 . The use of a single IC location tag is made possible by the simple signal structure at the core of the concept. Initially, only services that present either a significant increase in safety or a significant cost advantage over hospitalization or confinement alternatives will likely find widespread application. Two examples of important applications are addressed below. A s volume drives down per-unit costs, however, other applications such as pet location or routine cargo tracking will become economically feasible.

\section{LOCATING LOST PATENTS WITH ALZHEIMER's DISEASE}

All too often, newspapers report that someone with cognitive impairment has gotten lost and perished. There is a pressing need for the ability to quickly locate patients who have wandered off and become lost. In order to find the patient quickly, an accuracy of at least $100 \mathrm{ft}$ is desired.

The most common disease that can cause cognitive impairment and geriatric wandering is A Izheimer's disease. There is as yet no definitive diagnostic test in use and no cure for this disease, although much promising research is in progress. The disease is progressive, and patients experience increasing and irreversible levels of confusion, memory loss, personality change, behavior change, and impaired judgment. Patients inevitably lose orientation, and have difficulty remembering directions.

$D$ ata regarding the percentage of institutionalized dementia patients who wander range from 11 to 24 percent. In communitydwelling patients, studies have reported wandering in up to 59 percent of afflicted individuals. The goal of location management for wandering is not to totally confine the patients, because it is considered desirable to give patients both a sense of independence and the opportunity to exercise. $\mathrm{R}$ ather, the goal is to ensure the safety of the wandering individu$\mathrm{al}$, and to lessen the caregiver burden $[4,5]$.

Statistics indicate that currently over 4 million A mericans suffer from A lzheimer's disease. In 1989, it was estimated that over 10 percent of people over 65 were afflicted with the disease [6]. It has been estimated that nearly half ( 47 percent) of people aged 85 and older are afflicted with the disease [6]. With the progressive increase in the average age of the U.S. population, the number of A Izheimer's patients is predicted to increase at a rate of 10 percent/year. At such a rate, 14 million A mericans are expected to have the disease by the mid-21st century, unless a cure or means of prevention is found.

In the United States, over 75 percent of A lzheimer's patients, or over 3 million persons, live at home, in noninstitutional open surroundings. Most frequently, they are elderly but otherwise in good health and fully ambulant. Thus, they are very capable of wandering off. When they do, and it happens often, they are frequently unable to find their way back home. The situation is fraught with danger for the patients and causes considerable stress to a caregiver, often their spouse, who in general is also elderly.

A the present time, the only provision for such patients is a simple patient ID bracelet engraved with the patient's identity data, often including a number registered in a central registry. Such devices, while helpful, are very rudimentary, and rely on the patient being intercepted by, or encountering, somebody willing to take notice that he or she is a lost person.

L ocation services are considered most urgent for those who live at home, especially since the costs of institutionalization are high. $\mathrm{H}$ owever, even those who

Figure 3. L ow-cost low-power location unit.

Externally powered location unit: Fleet tracking Stolen car recovery Auto repossession 
live in community care facilities can benefit from such a device. The need arises because the facility may not be walled in and because residential facilities often permit residents freedom of movement within the facility. Such facilities seldom have sufficient staff to constantly monitor the whereabouts of each individual patient. A Izheimer's patients are generally not distinguishable by appearance from those whose memory is intact, and therefore their comings and goings do not evoke as much attention as those of patients who are visibly ill. A nursing home that loses a patient entrusted to its care is exposed to a very high liability risk.

A personal location system such as that required for A lzheimer's patients is shown in Fig. 4. By following the sequence of events, by either automatic detection or caregiver report, the patient is determined to be missing and the location system is invoked (1). The operations center (2) uses a conventional paging service (3) to activate the personal location unit (PLU), which activates and transmits a location signal (4). The surrounding receiver stations, coordinated via a terrestrial network, capture the PLU signal (5), perform preliminary processing, and send the reduced data to the center of operations (6). The center of operations calculates the position (7) and sends the result to the requesting person or emergency response agency (8), or to a computer for automated tracking applications such as parolee tracking, which is discussed below.

In ongoing work, we are defining the process by which emergency crews will use the location information to locate and recover the patient.

\section{Tracking Criminals}

A nother application STC is studying is the use of SLS for tracking and monitoring people in criminal justice applications.

In this case, the requirement is for a technique that can provide a wide-area low-cost radio location system for electronic monitoring and location of offenders who have been released into the community on parole, probation, or pretrial release. The need to solve this problem is well recognized [7], and this need becomes continuously more urgent as increasing numbers of individuals are being released from overcrowded detention centers, or are placed under observation for a long time under court orders while awaiting trial.

Electronic monitoring ( $E M$ ) is already being used for many correctional purposes, but existing systems are quite rudimentary. Current applications of EM devices include supervision of curfew confinements, home detention, and home incarceration monitoring. Such devices are sometimes called "first-generation" monitoring devices [7] and involve merely the detection of proximity of the offender to a fixed location or detection of perimeter crossings. In such perimeter detection systems, an alert (or alarm signal) is triggered when the individual crosses a threshold. The radio technology used is similar to what is commonly used in retail theft control systems and libraries for the checkout of books, but the prisoner monitoring system uses a secure bracelet which cannot readily be removed by the subject without triggering an alert.

The more important problem concerns electronic monitoring over a larger range, providing more freedom to the wearer of the device but also providing a record of the movements of the offender within the community. Such devices are known as "second-generation" monitoring devices. A $n$ alarm would be triggered when the person being monitored is not within a specified geographical area or approaches its preprogrammed limits. There is significant interest in such technology for probation and parole. In many cases, it can also be considered as an alternative to imprisonment $[8,9]$.

When electronic monitoring can be expanded to routinely include supervision of individuals restricted to stay within a larger specified area, it will become possible for some persons who now have to be confined in prison to instead stay outside the prison while being monitored electronically. U nfortunately, the existing technologies for position location have many drawbacks: they either are ineffective within buildings, have limited range of cov-

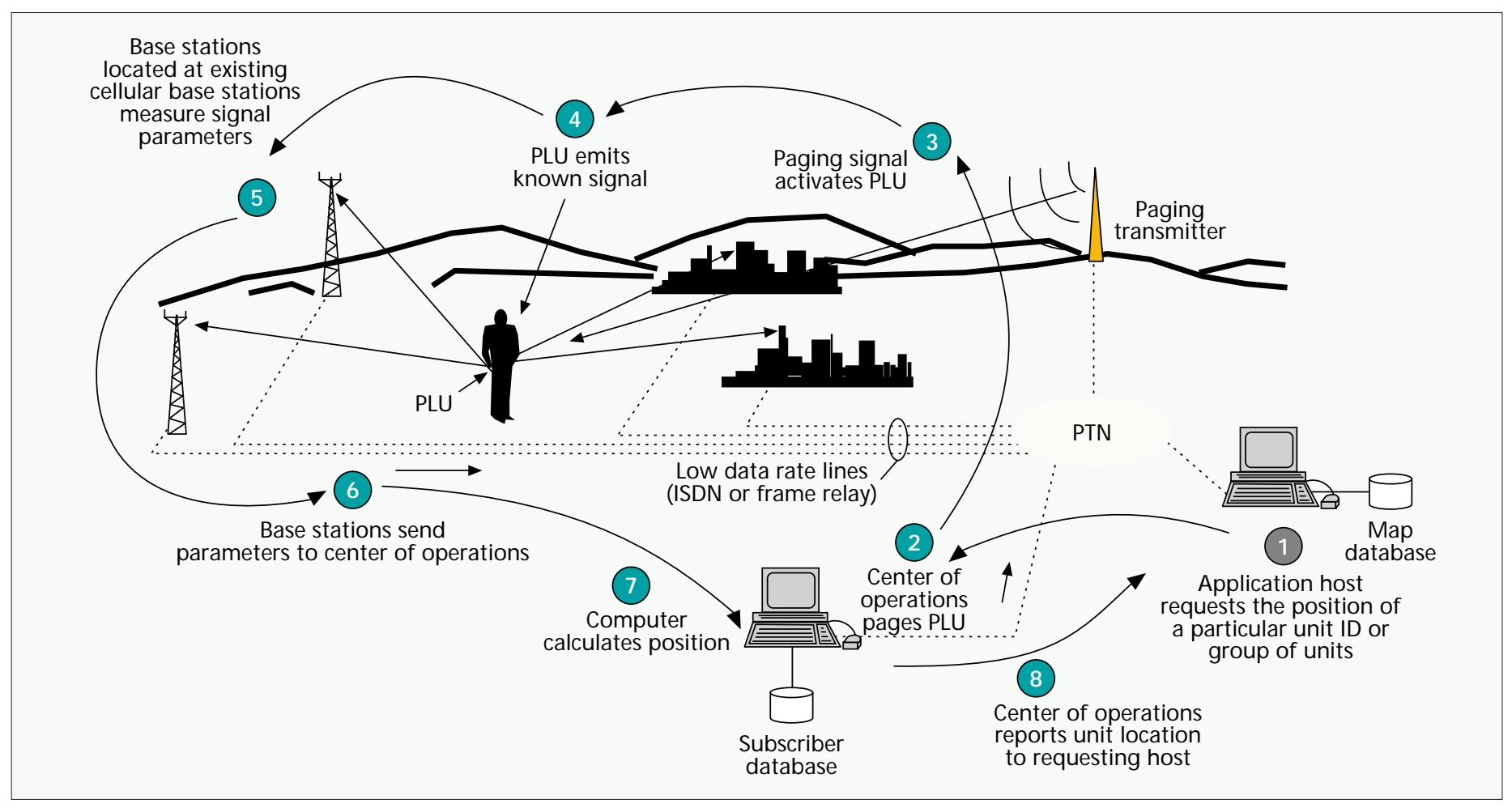

Figure 4. Person location/tracking using the SL S. 
erage or have poor accuracy, the unit carried by the individual being tracked is large; or the unit consumes so much power that it drains batteries too rapidly. The SLS may offer a possible solution to these problems.

It is likely that a position location system, such as SLS, will be combined with first-generation perimeter alarm systems, and designed to be activated, or deactivated, only when the individual crosses a specified boundary.

\section{ACKNOWLEDGMENTS}

This publication was made possible in part by grant number R 41A G 12573-01 from the N ational Institutes of $\mathrm{H}$ ealth $(\mathrm{NIH})$; award number 97-L B-V X-K 003 from the O ffice of J ustice Programs, National Institute of J ustice (NIJ), D epartment of J ustice; and grant number D M I-9560808 from the National Science F oundation (NSF). I ts contents are solely the responsibility of the authors and do not necessarily represent the official views of the $\mathrm{NIH}$, the U .S. Department of J ustice, or the NSF.

In addition we would like to thank J ay W eitzen and $\mathrm{H}$ aifeng Q iu for their valuable contributions to this publication.

\section{REFERENCES}

[1] Notice of Proposed Rulemaking, Docket 94-102, FCC, 1994.

[2] “Wireless Location Senvices: 1997," Strategis Group, 1997.

[3] T. S. Rappaport, J. H. Reed, and B. D. Woerner, "Position Location Using Wireless Communications on Highways of the Future," IEEE Commun. Mag., Oct. 1996, pp. 33-41.

[4] K. D. Melillo and M. Futrell, “Wandering: Survey of Formal Care Providers and Informal Caregivers Regarding the Use of a Low-Cost Patient Locator Unit," submitted to J. Gerontological Nursing, 1997.

[5] L. Morishita, "Wandering Behavior," Alzheimer's Disease: Treatment and Long-Term Management, Cummings and Miller, Eds. 1990, New York: Marcel Dekker, pp. 157-76.
[6] D. A. Evans et al., "Prevalence of Alzheimer's Disease in a Community Population of Older Persons," J. AMA, vol. 18, 1989, pp. 2551-56.

[7] J. Hoshen, J. Sennott and M. Winkler, "Keeping Tabs on Criminals," IEEE Spectrum, 1995, pp. 26-32.

[8] J. K. Stewart, E. W. Zedlewski, and L. E. Ray, "The Economics of Disincarceration," Selective Notification of Information, U.S. Dept. of Justice, Nat'I. Inst. of Justice/NCJRS, NIJ Reports/SNI 185, vol. May, pp. 19-84.

[9] P. J. P. Tak, “Alternatives to Imprisonment: International Summaries, Nat'l. Inst. of Justice/NCJRS, NCJ 114272.

\section{BIOGRAPHIES}

JAMES M. ZAGAMI (sig@world.std.com) is senior signal processing engineer at Signatron Technology Corporation. He received his B.S. in electrical engineering from the University of Massachusetts at Lowell in 1990. He currently has a patent pending involving radio location.

StEEn A. PARL (sig@world.std.com) is president of Signatron Technology Corporation. He received his Ph.D. in electrical engineering from the Massachusetts Institute of Technology in 1974, and his M.S. degree in electrical engineering from the Technical University of Denmark in January, 1970. He has published approximately 40 papers and holds five patents, with another patent pending.

JULIAN J. BuSSGANG (jjbussgang@aol.com) founded Signatron, Inc. (predecessor of Signatron Technology Corporation) in 1962 and is a highly regarded communications expert. He received his Ph.D. in applied physics from Harvard University in 1955, and his M.S. in electrical engineering from the Massachusetts Institute of Technology in 1951. He holds several patents and has published numerous papers.

KAREN DeVEREAux MeLLLo (karen_melillo@uml.edu) is a professor in the Department of Nursing at the University of Massachusetts Lowell. She received her Ph.D. from Brandeis University in 1990, and her M.S. in gerontological nursing from the University of Massachusetts at Lowell in 1978. She is published in many professional journals, and has served as principal investigator for several grants and contracts. 